\title{
ON THE SOLUTION OF PROBLEMS OF DYNAMIC PLANE ELASTICITY*
}

\author{
BY \\ J. R. M. RADOK** \\ Aeronautical Research Laboratories, Melbourne, Australia
}

Application of complex variable methods to the dynamic equations of the plane theory of elasticity is shown to lead to a similarly lucid presentation of boundary value problems as in the case of the static equations. A stress function may be introduced, which is shown to be a solution of a fourth order partial differential equation which reduces to a generalized biharmonic equation of the type occurring in anisotropic elasticity, when it is required to find plane wave solutions. This stress function can then be expressed in terms of two analytic functions of two complex variables and the stress and displacement components may be found in terms of these functions.

Three problems are solved by this method to illustrate its advantages: the moving Griffith crack, punch and dislocations.

1. Introduction. The introduction of the theory of functions of a complex variable into static plane elasticity of isotropic media [1] has considerably widened the scope for solving specific problems in this field. The reason for this development is to be found in the replacement of the indirect approach to the solution of boundary value problems by a direct one. In the present paper an attempt has been made to apply similar reasoning to the dynamic equations of plane elasticity.

The main difference between the methods of this report and those of [1] arises from the fact that the stress function here has to satisfy a generalized biharmonic equation which is of the same type as that occurring in the plane theory of elasticity of anisotropic media. S. G. Lekhnitzki [2] first introduced the theory of functions of a complex variable into this subject and showed that the stress function can be represented in terms of two analytic functions of one of two different complex variables each.

Some of the results obtained below were deduced by different means in 1937 by E. C. Titchmarsh [3] when studying the theory of Hilbert transforms.

2. The basic equations. In the case of plane elasticity the equations of motion are

$$
\begin{aligned}
& \frac{\partial \sigma_{x}}{\partial x}+\frac{\partial \tau_{x y}}{\partial y}=\rho \frac{\partial^{2} u}{\partial t^{2}}, \\
& \frac{\partial \tau_{x y}}{\partial x}+\frac{\partial \sigma_{y}}{\partial y}=\rho \frac{\partial^{2} v}{\partial t^{2}} .
\end{aligned}
$$

The stress-strain relations are the same as in the case of static elasticity

$$
\begin{aligned}
\sigma_{x}=\lambda \theta+2 \mu \frac{\partial u}{\partial x}, & \sigma_{\nu} & =\lambda \theta+2 \mu \frac{\partial v}{\partial y}, \\
\tau_{x y}=\mu\left(\frac{\partial u}{\partial y}+\frac{\partial v}{\partial x}\right), & \theta & =\frac{\partial u}{\partial x}+\frac{\partial v}{\partial y} .
\end{aligned}
$$

*Received Oct. 31, 1955.

**Now at Brown University, Providence, R. I. 
The first two of these may be rewritten in a form which will be found useful later on

$$
\begin{gathered}
2 \mu \frac{\partial u}{\partial x}=\sigma_{x}-\frac{\lambda}{2(\lambda+\mu)}\left(\sigma_{x}+\sigma_{y}\right), \quad 2 \mu \frac{\partial v}{\partial y}=\sigma_{y}-\frac{\lambda}{2(\lambda+\mu)}\left(\sigma_{x}+\sigma_{y}\right), \\
\theta=\frac{1}{2(\lambda+\mu)}\left(\sigma_{x}+\sigma_{y}\right) .
\end{gathered}
$$

Finally, the equation of compatibility must be satisfied

$$
\left\{\frac{\partial^{2}}{\partial x^{2}}+\frac{\partial^{2}}{\partial y^{2}}\right\}\left\{\sigma_{x}+\sigma_{y}\right\}-\frac{2(\lambda+\mu)}{\lambda+2 \mu} \rho \frac{\partial^{2}}{\partial t^{2}}\left(\frac{\partial u}{\partial x}+\frac{\partial v}{\partial y}\right)=0 ;
$$

using (2.3), this may be rewritten

$$
\left\{\frac{\partial^{2}}{\partial x^{2}}+\frac{\partial^{2}}{\partial y^{2}}-\frac{\rho}{\lambda+2 \mu} \frac{\partial^{2}}{\partial t^{2}}\right\}\left\{\sigma_{x}+\sigma_{\nu}\right\}=0 .
$$

Differentiating (2.1) with respect to $x$ and $y$ respectively and adding one finds by (2.2) and (2.3)

$$
\begin{gathered}
\frac{\partial^{2} \sigma_{x}}{\partial x^{2}}-\frac{\partial^{2} \sigma_{y}}{\partial y^{2}}=\frac{\rho}{2 \mu} \frac{\partial^{2}}{\partial t^{2}}\left(\sigma_{x}-\sigma_{y}\right), \\
\frac{\partial^{2}}{\partial x \partial y}\left(\sigma_{x}+\sigma_{y}\right)+\left(\frac{\partial^{2}}{\partial x^{2}}+\frac{\partial^{2}}{\partial y^{2}}-\frac{\rho}{\mu} \frac{\partial^{2}}{\partial t^{2}}\right) \tau_{x y}=0 .
\end{gathered}
$$

The complex representation of a certain type of solution of Eqs. $\left(2.4^{\prime}\right)-(2.6)$ is the object of the next section.

3. The stress function and its complex representation. Equation (2.5) may be rewritten

$$
\left\{\frac{\partial^{2}}{\partial x^{2}}-\frac{\rho}{2 \mu} \frac{\partial^{2}}{\partial t^{2}}\right\} \sigma_{x}=\left\{\frac{\partial^{2}}{\partial y^{2}}-\frac{\rho}{2 \mu} \frac{\partial^{2}}{\partial t^{2}}\right\} \sigma_{\nu}
$$

and it is seen that this equation will be satisfied by a stress function $U$ defined in the following manner:

$$
\sigma_{x}=\left\{\frac{\partial^{2}}{\partial y^{2}}-\frac{\rho}{2 \mu} \frac{\partial^{2}}{\partial t^{2}}\right\} U, \quad \sigma_{\nu}=\left\{\frac{\partial^{2}}{\partial x^{2}}-\frac{\rho}{2 \mu} \frac{\partial^{2}}{\partial t^{2}}\right\} U .
$$

Using (3.2) one has

$$
\sigma_{x}+\sigma_{y}=\left\{\frac{\partial^{2}}{\partial x^{2}}+\frac{\partial^{2}}{\partial y^{2}}-\frac{\rho}{\mu} \frac{\partial^{2}}{\partial t^{2}}\right\} U
$$

and (2.6) and $\left(2.4^{\prime}\right)$ become

$$
\begin{gathered}
\left\{\frac{\partial^{2}}{\partial x^{2}}+\frac{\partial^{2}}{\partial y^{2}}-\frac{\rho}{\mu} \frac{\partial^{2}}{\partial t^{2}}\right\}\left\{\tau_{x y}+\frac{\partial^{2} U}{\partial x \partial y}\right\}=0, \\
\left\{\frac{\partial^{2}}{\partial x^{2}}+\frac{\partial^{2}}{\partial y^{2}}-\frac{\rho}{\lambda+2 \mu} \frac{\partial^{2}}{\partial t^{2}}\right\}\left\{\frac{\partial^{2}}{\partial x}+\frac{\partial^{2}}{\partial y^{2}}-\frac{\rho}{\mu} \frac{\partial^{2}}{\partial t^{2}}\right\} U=0 .
\end{gathered}
$$

It is thus seen that $U$ must be the solution of a generalized biharmonic equation, formed by the product of two differential wave operators. The sum of the shear stress $\tau_{x y}$ and the mixed second derivative $\partial^{2} U / \partial x \partial y$ must also satisfy a wave equation. It is 
clear that in the case when the time $t$ is not a variable, the function $U$ becomes Airy's stress function.

In what follows attention will be restricted to problems for which the following transformation holds true

$$
\xi=x \pm c t, \quad \eta=y
$$

such a transformation corresponds to the occurrence of disturbances moving with velocity $c$ parallel to the axis $O x$. It is obvious that a more general approach could be adopted by use of the transformation

$$
\xi=x \pm c_{1} t, \quad \eta=y \pm c_{2} t .
$$

However, this has not been done here because it tends to complicate the following equations without introducing any basically new aspects; in particular, a rotation of the system of axes about the origin would lead to the same results. Using (3.6) Eq. (3.5) becomes

$$
\left\{\frac{\partial^{2}}{\partial \xi^{2}}\left(1-\frac{\rho c^{2}}{\lambda+2 \mu}\right)+\frac{\partial^{2}}{\partial \eta^{2}}\right\}\left\{\frac{\partial^{2}}{\partial \xi^{2}}\left(1-\frac{\rho c^{2}}{\mu}\right)+\frac{\partial^{2}}{\partial \eta^{2}}\right\} U=0
$$

with the real solution

where

$$
U=F_{1}\left(z_{1}\right)+F_{2}\left(z_{2}\right)+\overline{F_{1}}\left(\bar{z}_{1}\right)+\overline{F_{2}}\left(\bar{z}_{2}\right)
$$

$$
z_{1}=\xi+s_{1} \eta, \quad z_{2}=\xi+s_{2} \eta,
$$

$s_{i}$ being the roots of the characteristic equation of (3.7)

i.e.,

$$
\left.\left\{1-\frac{\rho c^{2}}{\lambda+2 \mu}+s^{2}\right\} 1-\frac{\rho c^{2}}{\mu}+s^{2}\right\}=0,
$$

$$
s_{1}=i\left(1-\frac{\rho c^{2}}{\lambda+2 \mu}\right)^{1 / 2}=i \beta_{1}, \quad s_{2}=i\left(1-\frac{\rho c^{2}}{\mu}\right)^{1 / 2}=i \beta_{2} .
$$

The functions $F_{i}\left(z_{i}\right)$ are analytic functions of the complex variables $z_{i}$ and $\bar{F}_{i}\left(\bar{z}_{i}\right)$ are the corresponding conjugate functions.

It is now possible to express stresses and displacements in terms of derivatives of the functions $F_{i}\left(z_{i}\right)$ and hence to reduce boundary value problems of dynamic elasticity to problems of complex function theory.

4. Stresses, displacements and boundary value problems. In order to simplify the later results, introduce the notation

$$
\varphi=\frac{d F_{1}}{d z_{1}}, \quad \varphi^{\prime}=\frac{d \varphi}{d z_{1}}, \quad \psi=\frac{d F_{2}}{d z_{2}}, \quad \text { etc. }
$$

Then, by (3.6), (3.8) and (3.9),

$$
\begin{aligned}
& \frac{\partial^{2} U}{\partial \xi^{2}}=2 \operatorname{Re}\left\{\varphi^{\prime}+\psi^{\prime}\right\} \\
& \frac{\partial^{2} U}{\partial \eta^{2}}=2 \operatorname{Re}\left\{s_{1}^{2} \varphi^{\prime}+s_{2}^{2} \psi^{\prime}\right\}=-2 \operatorname{Re}\left\{\beta_{1}^{2} \varphi^{\prime}+\beta_{2}^{2} \psi^{\prime}\right\}, \\
& \frac{\partial^{2} U}{\partial t^{2}}=c^{2} \frac{\partial^{2} U}{\partial \xi^{2}}
\end{aligned}
$$


while (3.2) take the form

$$
\begin{aligned}
& \sigma_{y}=\left(1-\frac{\rho c^{2}}{2 \mu}\right) \frac{\partial^{2}}{\partial \xi^{2}} U=\frac{1}{2}\left(1+\beta_{2}^{2}\right) \frac{\partial^{2}}{\partial \xi^{2}} U, \\
& \sigma_{x}=\left\{\frac{\partial^{2}}{\partial \eta^{2}}-\frac{\rho c^{2}}{2 \mu} \frac{\partial^{2}}{\partial \xi^{2}}\right\} U .
\end{aligned}
$$

Hence one finds the following expressions for the stresses and displacements:

$$
\begin{aligned}
\sigma_{y} & =\left(1+\beta_{2}^{2}\right) \operatorname{Re}\left(\varphi^{\prime}+\psi^{\prime}\right), \\
\sigma_{x} & =-2 \operatorname{Re}\left\{\left[\beta_{1}^{2}+\frac{1}{2}\left(1-\beta_{2}^{2}\right)\right] \varphi^{\prime}+\frac{1}{2}\left[1+\beta_{2}^{2}\right] \psi^{\prime}\right\}, \\
\sigma_{x}+\sigma_{y} & =-2 \operatorname{Re}\left\{\left(\beta_{1}^{2}-\beta_{2}^{2}\right) \varphi^{\prime}\right\}, \\
\tau_{x y} & =-2 \operatorname{Re} i\left\{\beta_{1} \varphi^{\prime}+\frac{1}{4} \frac{\left(1+\beta_{2}^{2}\right)^{2}}{\beta_{2}} \psi^{\prime}\right\}, \\
u & =-\frac{\operatorname{Re}}{\mu}\left\{\varphi+\frac{1}{2}\left(1+\beta_{2}^{2}\right) \psi\right\}, \\
v & =-\frac{\operatorname{Re}}{\mu} i\left\{\beta_{1} \varphi+\frac{1}{2} \frac{\left(1+\beta_{2}^{2}\right)}{\beta_{2}} \psi\right\},
\end{aligned}
$$

where the last two have been obtained from (2.3) using the first three and integrations, while the expression for the shear stress $\tau_{x y}$ follows from the displacements by means of differentiations. In this work certain constants and linear terms have been omitted, since they only refer to rigid body motion.

It is readily seen that the formulae (4.4) satisfy the differential equations of Sec. 2. They have been deduced previously by Titchmarsh [3] when studying Hilbert transforms, using entirely different methods. Nevertheless, they have not, to the author's knowledge, been used for the reduction of boundary value problems of dynamic elasticity to problems of complex function theory.

Certain types of boundary value problems of dynamic elasticity have been discussed in detail in Chap. 3 of Ref. [1], and it is shown there that the solutions are unique. In general, boundary value problems will be stated in physical terms and their reduction to function theory by means of the formulae will present no difficulties. As in the static theory of isotropic media full use can be made of conformal mapping, except that, just as in anisotropic theory, the occurrence of the two variables $z_{i}$ requires the use of two mapping functions. In fact, it is obvious that a boundary $L$ in the $z$-plane, the original plane, will be distorted in the $z_{1}$ and $z_{2}$ planes. The statement of the boundary value problems in terms of two functions of $z_{1}$ and $z_{2}$ respectively therefore will require conformal transformations from the $z_{1}$ and $z_{2}$ planes on to the circle $\gamma$ in the $\zeta$ plane, where only one variable occurs.

These remarks will be further elucidated in the next section which deals with a fundamental problem of the type $I$ (stresses given on the boundary)-the problem of a finite length crack moving through a solid. This problem, solved by different means by $\mathrm{E}$. Yoffe [4], is of some interest in micro-elastic work. Another problem, readily treated by this method, is that of a parabolic punch moving along the boundary of a halfplane. This problem is solved in Sec. 6 . The problem of moving dislocations is briefly discussed in Sec. 7. 
5. The moving Griffith crack. Consider the case where uniform tension $p$ acts at infinity at right angles to the direction of the slot, extending in the $\xi, \eta$ plane from $-a$ to $+a$. The boundary conditions for this problem have the form

$$
\begin{array}{rlrl}
\sigma_{y} & =0, & & |\xi|<a \\
\tau_{x y} & =0 & & \\
\sigma_{y}^{(\infty)} & =p, & \sigma_{x}^{(\infty)}=0 .
\end{array}
$$

Using (4.4), one finds from these conditions

$$
\begin{gathered}
\operatorname{Re}\left\{\varphi^{\prime}\left(z_{1}\right)+\psi^{\prime}\left(z_{2}\right)\right\}=0, \\
-2 \operatorname{Re} i\left\{\beta_{1} \varphi^{\prime}\left(z_{1}\right)+\frac{\left(1+\beta_{2}^{2}\right)^{2}}{4 \beta_{2}} \psi^{\prime}\left(z_{2}\right)\right\}=0,
\end{gathered}
$$

and the problem is to find analytic functions $\varphi\left(z_{1}\right), \psi\left(z_{2}\right)$ which give the required stresses at infinity and satisfy these boundary conditions.

Obviously the uniform loading at infinity will be caused by linear terms in $\varphi$ and $\psi$; therefore one may write

$$
\varphi\left(z_{1}\right)=\left(B^{*}+C^{*}\right) z_{1}+\varphi_{0}\left(z_{1}\right), \quad \psi\left(z_{2}\right)=\left(B^{* \prime}+C^{* \prime}\right) z_{2}+\psi_{0}\left(z_{2}\right)
$$

and express the constants $B^{*}, C^{*}, B^{* \prime}, C^{* \prime}$ in terms of the loading at infinity. By (4.4), one finds

$$
\begin{gathered}
B^{*}=-\frac{\sigma_{x}^{(\infty)}+\sigma_{\nu}^{(\infty)}}{2\left(\beta_{1}^{2}-\beta_{2}^{2}\right)}, \quad B^{* \prime}=\frac{\sigma_{\nu}^{(\infty)}}{1+\beta_{2}^{2}}+\frac{\sigma_{x}^{(\infty)}+\sigma_{\nu}^{(\infty)}}{2\left(\beta_{1}^{2}-\beta_{2}^{2}\right)}, \\
C^{*}=\frac{\tau_{x y}^{(\infty)}}{2 \beta_{1}}, \quad C^{* \prime}=0 .
\end{gathered}
$$

Substituting (5.3) in (5.2) the modified boundary conditions become, since $C^{* \prime}=0$,

$$
\begin{gathered}
\operatorname{Re}\left\{\varphi_{0}^{\prime}\left(z_{1}\right)+\psi_{0}^{\prime}\left(z_{2}\right)\right\}=-\left(B^{*}+B^{* \prime}\right), \\
\operatorname{Re} i\left\{\beta_{1} \varphi_{0}^{\prime}\left(z_{1}\right)+\frac{\left(1+\beta_{2}^{2}\right)^{2}}{4 \beta_{2}} \psi_{0}^{\prime}\left(z_{2}\right)\right\}=\beta_{1} C^{*} .
\end{gathered}
$$

Since in the present problem the boundary is part of the real axis, it is the same in the $z, z_{1}, z_{2}$ planes and the mapping function on to the unit circle is

$$
\frac{z_{1,2}}{a}=\frac{1}{2}\left(\zeta+\frac{1}{\zeta}\right)
$$

so that

$$
a \zeta=z_{1,2}-\left(z_{1,2}^{2}-a^{2}\right)^{1 / 2} .
$$

Normalizing and transforming (5.5) and using the well-known result of function theory that an analytic function whose boundary value is constant is a constant throughout its range of definition, one finds

$$
\begin{gathered}
\varphi_{0}^{\prime}(\zeta)+\psi_{0}^{\prime}(\zeta)=-a\left(B^{*}+B^{* \prime}\right), \\
i\left\{\beta_{1} \varphi_{0}^{\prime}(\zeta)+\frac{1}{4} \frac{\left(1+\beta_{2}^{2}\right)^{2}}{\beta_{2}} \psi_{0}^{\prime}(\zeta)\right\}=a \beta C^{*} .
\end{gathered}
$$


Consider now the special case

$$
\sigma_{\nu}^{(\infty)}=p, \quad \sigma_{x}^{(\infty)}=\tau_{x y}^{(\infty)}=0,
$$

i.e.,

$$
\begin{gathered}
B^{*}=-\frac{p}{2\left(\beta_{1}^{2}-\beta_{2}^{2}\right)}, \quad B^{* \prime}=\frac{p}{1+\beta_{2}^{2}}+\frac{p}{2\left(\beta_{1}^{2}-\beta_{2}^{2}\right)}, \\
C^{*}=0 .
\end{gathered}
$$

Then, from (5.8),

$$
\varphi_{0}^{\prime}(\zeta)=-\frac{a p\left(1+\beta_{2}^{2}\right)}{\left(1+\beta_{2}^{2}\right)^{2}-4 \beta_{1} \beta_{2}}, \quad \psi_{0}^{\prime}(\zeta)=\frac{4 \beta_{1} \beta_{2} p a}{\left(1+\beta_{2}^{2}\right)\left[\left(1+\beta_{2}^{2}\right)^{2}-4 \beta_{1} \beta_{2}\right]}
$$

and, after integrating with respect to $\zeta$ and using (5.7),

$$
\begin{aligned}
& \varphi_{0}\left(z_{1}\right)=-\frac{p\left(1+\beta_{2}^{2}\right)}{\left(1+\beta_{2}^{2}\right)^{2}-4 \beta_{1} \beta_{2}}\left\{z_{1}-\left(z_{1}^{2}-a^{2}\right)^{1 / 2}\right\} \\
& \psi_{0}\left(z_{2}\right)=\frac{4 \beta_{1} \beta_{2} p}{\left(1+\beta_{2}^{2}\right)\left[\left(1+\beta_{2}^{2}\right)^{2}-4 \beta_{1} \beta_{2}\right]}\left\{z_{2}-\left(z_{2}^{2}-a^{2}\right)^{1 / 2}\right\} .
\end{aligned}
$$

The expressions for $\varphi\left(z_{1}\right)$ and $\psi\left(z_{2}\right)$ may now be written down using (5.3). The stresses and displacements then follow from (4.4).

In order to check the solution, the stresses along the real axis will be calculated. By (4.4), the stress distribution is given by

$$
\begin{aligned}
& \sigma_{x}=\frac{p}{\left(1+\beta_{2}^{2}\right)^{2}-4 \beta_{1} \beta_{2}} \operatorname{Re}\left\{\left[2 \beta_{1}^{2}+1-\beta_{2}^{2}\right]\left[1+\beta_{2}^{2}\right]\left[1-\frac{z_{1}}{\left(z_{1}^{2}-a^{2}\right)^{1 / 2}}\right]\right. \\
& \left.-4 \beta_{1} \beta_{2}\left[1-\frac{z_{2}}{\left(z_{2}^{2}-a^{2}\right)^{1 / 2}}\right]\right\}, \\
& \sigma_{y}=\frac{\left(1+\beta_{2}^{2}\right) p}{\left(1+\beta_{2}^{2}\right)^{2}-4 \beta_{1} \beta_{2}} \operatorname{Re}\left\{\left(1+\beta_{2}^{2}\right) \frac{z_{1}}{\left(z_{1}^{2}-a^{2}\right)^{1 / 2}}-\frac{4 \beta_{1} \beta_{2}}{\left(1+\beta_{2}^{2}\right)} \frac{z_{2}}{\left(z_{2}^{2}-a^{2}\right)^{1 / 2}}\right\}, \\
& \tau_{x y}=\frac{-2 p\left(1+\beta_{2}^{2}\right) \beta_{1}}{\left(1+\beta_{2}^{2}\right)^{2}-4 \beta_{1} \beta_{2}} \operatorname{Re} i\left\{\frac{z_{1}}{\left(z_{1}^{2}-a^{2}\right)^{1 / 2}-\frac{z_{2}}{\left(z_{2}^{2}-a^{2}\right)^{1 / 2}}}\right\}
\end{aligned}
$$

and hence along the real axis

$$
\begin{aligned}
& \sigma_{x}=\frac{\left(2 \beta_{1}^{2}+1-\beta_{2}^{2}\right)\left(1+\beta_{2}^{2}\right)-4 \beta_{1} \beta_{2}}{\left(1+\beta_{2}^{2}\right)^{2}-4 \beta_{1} \beta_{2}}\left\{\begin{array}{cc}
p & \text { for }|\xi|<a, \\
\left(1-\frac{\xi}{\left(\xi-a^{2}\right)^{1 / 2}}\right) p & \text { for }|\xi|>a,
\end{array}\right. \\
& \sigma_{\nu}=\left\{\begin{array}{cc}
0 & \text { for }|\xi|<a, \\
\frac{\xi p}{\left(\xi^{2}-a^{2}\right)^{1 / 2}} & \text { for }|\xi|>a,
\end{array}\right. \\
& \tau_{x y}=0 \quad \text { for all } \xi \text {. }
\end{aligned}
$$

It is thus seen that the solution satisfies all the conditions of the problem. 
Next consider the transition to the static problem. By (3.11),

$$
\begin{aligned}
& \beta_{1}=\left(1-\frac{\rho c^{2}}{\lambda+2 \mu}\right)^{1 / 2}=1-\frac{\rho c^{2}}{2(\lambda+2 \mu)}+O\left(c^{4}\right), \\
& \beta_{2}=\left(1-\frac{\rho c^{2}}{\mu}\right)^{1 / 2}=1-\frac{\rho c^{2}}{2 \mu}+O\left(c^{4}\right),
\end{aligned}
$$

and therefore

$$
\lim _{c \rightarrow 0} \frac{\left(2 \beta_{1}^{2}+1-\beta_{2}^{2}\right)\left(1+\beta_{2}^{2}\right)-4 \beta_{1} \beta_{2}}{\left(1+\beta_{2}^{2}\right)^{2}-4 \beta_{1} \beta_{2}}=-1
$$

and (5.13) gives

$$
\begin{aligned}
& \sigma_{x}=\left\{\begin{array}{ccc}
-p & \text { for } & |x|<a, \\
-p\left(1-\frac{x}{\left(x^{2}-a^{2}\right)^{1 / 2}}\right) & \text { for } & |x|>a,
\end{array}\right. \\
& \sigma_{y}=\left\{\begin{array}{cc}
0 & \text { for }|x|<a \\
\frac{x p}{\left(x^{2}-a^{2}\right)^{1 / 2}} & \text { for }|x|>a,
\end{array}\right. \\
& \tau_{x y}=0 \quad \text { for all } x,
\end{aligned}
$$

which is the solution found by C. E. Inglis in 1913.

The expressions (5.13) agree fully with the results obtained by E. Yoffe [4] whose analysis and formulae are easily related to those of the present report when it is realized that in her notation

$$
f\left(z_{1}\right)=-\frac{i \varphi\left(z_{1}\right) \beta_{1}}{\mu}, \quad g\left(z_{2}\right)=-\frac{i\left(1+\beta_{2}^{2}\right)}{2 \mu} \psi\left(z_{2}\right), \quad \gamma=\beta_{1}, \quad \beta=\beta_{2} .
$$

The physical interpretation of the functions $\varphi, \psi$ is deduced from [4], which constructs them from simple wave solutions by means of Fourier integrals. It is thus seen that $\varphi, \psi$ are associated with longitudinal and shear waves respectively travelling through the medium with the velocities

$$
c_{1}=\left(\frac{\lambda+2 \mu}{\rho}\right)^{1 / 2}, \quad c_{2}=\left(\frac{\mu}{\rho}\right)^{1 / 2} .
$$

6. The parabolic punch moving over a half-plane. It will be assumed that no friction occurs. Let the profile of the rigid punch be given by

$$
f(x)=d-\frac{x^{2}}{2 R}, \quad R \text { large, }
$$

and assume that it is in contact with the elastic half-plane over the segment $|\xi| \leq l$ of the $\xi$ axis. Let the remaining part of the real axis be unloaded. Under these conditions the quasi-static boundary value problem takes the form

$$
\begin{aligned}
\tau_{x y} & =0 & & \text { for all points of the } \xi \text { axis, } \\
\sigma_{\nu} & =0 & & \text { for }|\xi|>l \\
v & =f(\xi) & & \text { for }|\xi| \leq l
\end{aligned}
$$


By (4.4) these conditions lead to

$$
\begin{gathered}
\beta_{1}\left\{\varphi^{\prime}\left(z_{1}\right)-\overline{\varphi^{\prime}}\left(\bar{z}_{1}\right)\right\}+\frac{1}{4} \frac{\left(1+\beta_{2}^{2}\right)^{2}}{\beta_{2}}\left\{\psi^{\prime}\left(z_{2}\right)-\overline{\psi^{\prime}}\left(\bar{z}_{2}\right)\right\}=0 \text { for all real } \xi, \\
\varphi^{\prime}\left(z_{1}\right)+\overline{\varphi^{\prime}}\left(\bar{z}_{1}\right)+\psi^{\prime}\left(z_{2}\right)+\overline{\psi^{\prime}}\left(\bar{z}_{2}\right)=0 \text { for }|\xi|>l, \\
\beta_{1}\left\{\varphi^{\prime}\left(z_{1}\right)-\overline{\varphi^{\prime}}\left(\bar{z}_{1}\right)\right\}+\frac{1}{2} \frac{\left(1+\beta_{2}^{2}\right)}{\beta_{2}}\left\{\psi^{\prime}\left(z_{2}\right)-\overline{\psi^{\prime}}\left(\bar{z}_{2}\right)\right\}=2 \mu i f^{\prime} \text { for }|\xi| \leq l,
\end{gathered}
$$

where the last equation has been obtained from (4.4) by differentiation with respect to $\xi$. These boundary conditions must be understood in the sense that they hold true for

$$
z_{1} \rightarrow \xi, \quad z_{2} \rightarrow \xi,
$$

from inside the upper half-plane $\eta>0$.

A solution of (6.3) will now be sought in the form

$$
\varphi\left(z_{1}\right)=A \chi\left(z_{1}\right), \quad \psi\left(z_{2}\right)=B \chi\left(z_{2}\right),
$$

where the initially arbitrary constants $A$ and $B$ are to be determined in such a way that the last two conditions (6.3) lead to a simple problem of linear relationship [1].

The first equation (6.3) gives then

$$
\left[A \beta_{1}+\frac{\beta}{4 \beta_{2}}\left(1+\beta_{2}^{2}\right)^{2}\right]\left[\chi^{\prime}-\bar{\chi}^{\prime}\right]=0
$$

for all real $\xi$, and hence

$$
A \beta_{1}+\frac{B}{4 \beta_{2}}\left(1+\beta_{2}^{2}\right)^{2}=0 .
$$

Similarily the last two conditions of (6.3) lead to

$$
(A+B)\left(\chi^{\prime}+\bar{\chi}^{\prime}\right)=0 \text { for }|\xi|>l,
$$

and

$$
\left[A \beta_{1}+\frac{B}{2 \beta_{2}}\left(1+\beta_{2}^{2}\right)\right]\left[\chi^{\prime}-\bar{\chi}^{\prime}\right]=2 \mu i f^{\prime} \text { for }|\xi| \leq l \text {. }
$$

The constants $A, B$ may now be determined from (6.5) and the condition

$$
A \beta_{1}+\frac{B}{2 \beta_{2}}\left(1+\beta_{2}^{2}\right)=1
$$

deduced from (6.7). One thus finds

$$
A=-\frac{1+\beta_{2}^{2}}{\beta_{1}\left(1-\beta_{2}^{2}\right)}, \quad B=\frac{4 \beta_{2}}{1-\beta_{2}^{4}} .
$$

Note that in the presence of friction (6.5) would no longer be valid.

It follows from (6.6) that the function $\chi$ will be holomorphic in the entire plane cut along $|\xi| \leq l$, if it is extended into the lower half-plane by

$$
\bar{\chi}(\bar{\zeta})=-\chi(\bar{\zeta}) \text {. }
$$


Then, as $\zeta \rightarrow \xi$ from $\eta>0, \bar{\zeta} \rightarrow \xi$ from $\eta<0$ and

$$
\chi(\zeta) \rightarrow \chi^{+}(\xi), \quad \chi(\bar{\zeta}) \rightarrow-\chi^{-}(\xi)
$$

Hence, using (6.8), (6.1) and (6.11), the boundary conditions (6.6) and (6.7) take the form

$$
\begin{aligned}
& \chi^{\prime+}-\chi^{\prime-}=0 \quad \text { for }|\xi|>l \text {, } \\
& \chi^{\prime+}+\chi^{\prime-}=-\frac{2 \mu i \xi}{R} \text { for }|\xi| \leq l \text {. }
\end{aligned}
$$

Using the Plemelj formulae [1] and taking into account that in view of the smoothness of the punch profile no singularities will occur, one has

$$
\begin{aligned}
\chi^{\prime}(\zeta) & \doteq \frac{-\mu\left(l^{2}-\zeta^{2}\right)^{1 / 2}}{R \pi} \int_{-l}^{+l} \frac{t d t}{(t-\zeta)\left(l^{2}-\zeta^{2}\right)^{1 / 2}} \\
& =\frac{\mu i}{R}\left\{\left(\zeta^{2}-l^{2}\right)^{1 / 2}-\zeta\right\}
\end{aligned}
$$

The solution of the problem follows now from (6.4) and (6.9):

$$
\begin{aligned}
& \varphi^{\prime}\left(z_{1}\right)=-\frac{\left(1+\beta_{2}^{2}\right)}{\left(1-\beta_{2}^{2}\right)} \frac{1}{\beta_{1}} \frac{i \mu}{R}\left\{\left(z_{1}^{2}-l^{2}\right)^{1 / 2}-z_{1}\right\}, \\
& \psi^{\prime}\left(z_{2}\right)=\frac{4 \beta_{2}}{\left(1-\beta_{2}^{4}\right)} \frac{i \mu}{R}\left\{\left(z_{2}^{2}-l^{2}\right)^{1 / 2}-z_{2}\right\} .
\end{aligned}
$$

The stresses are given by (4.4) and will here only be written down for the $\xi$ axis:

$$
\begin{aligned}
& \sigma_{\nu}=-\frac{4 \beta_{1} \beta_{2}-\left(1+\beta_{2}^{2}\right)^{2}}{\left(1-\beta_{2}^{2}\right)} \frac{\mu}{R \beta_{1}}\left(l^{2}-\xi^{2}\right)^{1 / 2} \quad \text { for } \quad|\xi| \leq l, \\
& \sigma_{x}=\frac{\left\{4 \beta_{1} \beta_{2}-\left(1+\beta_{2}^{2}\right)\left(1+2 \beta_{1}^{2}-\beta_{2}^{2}\right)\right\}}{\left(1-\beta_{2}^{2}\right)} \frac{\mu}{R \beta_{1}}\left(l^{2}-\xi^{2}\right)^{1 / 2} \text { for }|\xi| \leq l \text {. }
\end{aligned}
$$

The total force acting on the punch is

$$
Y=\int_{-l}^{+l} \sigma_{\nu} d \xi=-\frac{\left\{4 \beta_{1} \beta_{2}-\left(1+\beta_{2}^{2}\right)^{2}\right\}}{\left(1-\beta_{2}^{2}\right)} \frac{\mu}{R \beta_{1}} \frac{l^{2} \pi}{2} .
$$

When the velocity $c$ with which the punch moves in the direction $\xi$ tends to zero, the above results reduce to those of the static theory, as was to be expected.

7. Moving dislocation. In [5] the author looks for a solution of the differential equations of Sec. 2 which is to satisfy the following conditions:

$$
\begin{aligned}
\sigma_{\nu} & =0, \quad \text { for } \quad \eta=0 \\
{[u]_{L} } & =\text { const. }
\end{aligned}
$$

where []$_{L}$ denotes the change undergone by the quantity inside the brackets for one circuit of $L$. It is easily seen from (4.4) that it follows from the first of these conditions that

$$
\varphi(\xi)=-\psi(\xi)
$$


while the second is satisfied by the functions

$$
\varphi=b \log z_{1}, \quad \psi=-b \log z_{2},
$$

which satisfy also the first condition. Hence, by the last two expressions of (4.4),

$$
\begin{aligned}
& u=-\frac{R e}{\mu} b\left\{\log z_{1}-\frac{1}{2}\left(1+\beta_{2}^{2}\right) \log z_{2}\right\}, \\
& v=-\frac{R e}{\mu} i b\left\{\beta_{1} \log z_{1}-\frac{1}{2} \frac{\left(1+\beta_{2}^{2}\right)^{2}}{\beta_{2}} \log z_{2}\right\},
\end{aligned}
$$

and, since

$$
\log z=\log (x+i y)=\log \left(x^{2}+y^{2}\right)^{1 / 2}+i \operatorname{artan} y / x,
$$

the displacements (7.1) agree with those given by formulae (12) and (13) of [5].

In the work of this section, as also elsewhere in this paper, integration constants have been omitted.

8. Conclusions. The introduction of the complex variable approach into the study of the dynamic equations of plane elasticity leads to a simplification of the statement as well as of the process of solution of problems dealing with surface waves. In fact, the relevant solution of the differential equations is found to be expressible in terms of two analytic functions, corresponding to the two types of waves, occurring in elastic media. As in static elasticity, the stress and displacement components are easily expressed in terms of these two functions and in this way boundary value problems can be reduced to problems of complex function theory.

The method has been applied to three problems some of which were previously solved by other more complicated methods: the moving Griffith crack [4], the moving punch and moving dislocations [5].

Not added in proof. Since completion of this paper the author has discovered that the problem of the moving punch has been solved by a similar method in [6].

\section{REFERENCES}

1. N. I. Muskhelishvili, Some basic problems of the mathematical theory of elasticity, N. V. Erven P. Noordhoff, Groningen, 1953

2. S. .G Lekhnitzki, Anisotropic plates, OGIS, Moscow, 1947

3. E. C. Titchmarsh, Introduction to the theory of Fourier integrals, Oxford Press, 1937

4. Elizabeth Yoffe, The moving Griffith crack, Phil. Mag., 42, No. 330, July 1951

5. J. D. Eshelby, Uniformly moving dislocations, Proc. Phys. Soc., 62, part 5, No. 353A, 1949

6. L. A. Galin, The contact problems of the theory of elasticity, Gostechizdat, 1953 